\title{
Endoscopic Diagnosis of Nonpedunculated Dysplasia during Surveillance of Ulcerative Colitis: A Survey-Based Multinational Study
}

\begin{abstract}
Dong-Hoon Yang ${ }^{1}$, Sneha John ${ }^{2}$, Fujishiro Mitsuhiro ${ }^{3}$, Jae Myung Cha ${ }^{4}$, Jeong-Sik Byeon ${ }^{1}$, Hyung Wook Kim ${ }^{5}$, Hyun Gun $\mathrm{Kim}^{6}$, Shai Friedland ${ }^{7,8}$, Yon Xian Koh ${ }^{2}$, Jin-Young Yoon ${ }^{4}$, Min-Seob Kwak ${ }^{4}$, Byong Duk Ye ${ }^{1,9}$, Jihun Kim ${ }^{10}$, and Suk-Kyun Yang $^{1,9}$

${ }^{1}$ Department of Gastroenterology, Asan Medical Center, University of Ulsan College of Medicine, Seoul, Korea, ${ }^{2}$ Department of Gastroenterology, Gold Coast University Hospital, Southport, Australia, ${ }^{3}$ Department of Gastroenterology, Nagoya University Graduate School of Medicine, Nagoya, Japan, ${ }^{4}$ Department of Internal Medicine, Kyung Hee University Hospital at Gangdong, Kyung Hee University School of Medicine, Seoul, ${ }^{5}$ Division of Gastroenterology, Department of Internal Medicine, Pusan National University School of Medicine and Medical Research Institute, Pusan National University Yangsan Hospital, Yangsan, ${ }^{6}$ Department of Gastroenterology, Soonchunhyang University College of Medicine, Seoul, Korea, 'Department of Gastroenterology and Hepatology, Stanford University School of Medicine, Stanford, CA, ${ }^{8}$ Department of Gastroenterology, VA Palo Alto Health Care System, Palo Alto, CA, USA, ${ }^{9}$ Inflammatory Bowel Disease Center, and ${ }^{10}$ Department of Pathology, Asan Medical Center, University of Ulsan College of Medicine, Seoul, Korea
\end{abstract}

Background/Aims: Endoscopic diagnosis of dysplasia or colitic cancer in patients with ulcerative colitis (UC) is more challenging than that of colorectal neoplasia in non-colitic patients. We aimed to evaluate the accuracy of the endoscopic diagnosis of "nonpedunculated" dysplasia or colitic cancer in UC patients. Methods: Ten endoscopists from four countries were surveyed using photographs of 61 histologically confirmed dysplastic or non-dysplastic lesions retrieved from the UC registry database of Asan Medical Center. The participants provided their assessment based on the given photographs and their intention to perform biopsy. Results: The overall diagnostic performance of the 10 participants is summarized as follows: sensitivity of $88.2 \%$ (95\% confidence interval [Cl], $84.3 \%$ to $91.5 \%)$, specificity of $34.8 \%(95 \% \mathrm{Cl}$, $29.1 \%$ to $40.8 \%$ ), positive predictive value of $63.0 \%$ (95\% $\mathrm{Cl}, 60.8 \%$ to $65.2 \%$ ), negative predictive value of $70.2 \%$ (95\% Cl, $62.7 \%$ to $76.6 \%)$, and accuracy of $64.6 \%(95 \% \mathrm{Cl}$, $60.7 \%$ to $68.4 \%$ ). The interobserver agreement on the intention to perform a biopsy was poor (Fleiss kappa=0.169). Of the three endoscopic characteristics of the lesions, including ulceration, distinctness of the borders, and pit patterns, only neoplastic pit patterns were significantly predictive of dysplasia (odds ratio, 3.710; 95\% Cl, 2.001 to 6.881). The diagnostic sensitivity and specificity of neoplastic pit patterns were $68.2 \%$ (95\% Cl, 63.0\% to $73.2 \%$ ) and $63.3 \%(95 \% \mathrm{Cl}$, $57.3 \%$ to $69.1 \%)$, respectively. Conclusions: Diagnostic per- formance based on the endoscopist's intention to perform a biopsy for nonpedunculated potentially dysplastic lesions in UC patients was suboptimal according to this survey-based study. (Gut Liver 2020;14:611-618)

Key Words: Colitis, ulcerative; Dysplasia; Neoplasm, colonic; Colonoscopy; Diagnosis

\section{INTRODUCTION}

Long-standing ulcerative colitis (UC) patients are exposed to an increased risk of colorectal cancer (CRC), ${ }^{1,2}$ and thus need regular and risk-adjusted surveillance colonoscopy. ${ }^{3-5}$ The aim of surveillance colonoscopy in UC is to detect dysplasia or CRC at an early stage, and the timely detection of dysplasia or CRC may reduce CRC-related morbidity and mortality, or even prevent CRC through the resection of dysplasia. ${ }^{3}$ Previous studies suggested that chromoendoscopy (CE)-based targeted biopsy was more effective for the detection of dysplasia than traditional random biopsy. ${ }^{6-9}$ Therefore, CE-based targeted biopsy should be the primary surveillance technique, provided that appropriate CE expertise is available., ${ }^{3,10}$

Recognition of suspected dysplasia is the first step of the targeted biopsy strategy, with the endoscopic discrimination of potential dysplasia from definitely non-dysplastic lesions being the next step. In the earliest study evaluating the role of

\footnotetext{
Correspondence to: Dong-Hoon Yang

Department of Gastroenterology, Asan Medical Center, University of Ulsan College of Medicine, 88 Olympic-ro 43-gil, Songpa-gu, Seoul 05505, Korea
}

Tel: +82-2-3010-5809, Fax: +82-2-3010-6517, E-mail: dhyang@amc.seoul.kr

Received on July 11, 2019. Revised on September 5, 2019. Accepted on September 23, 2019. Published online December 18, 2019. pISSN 1976-2283 eISSN 2005-1212 https://doi.org/10.5009/gnl19237

(a) This is an Open Access article distributed under the terms of the Creative Commons Attribution Non-Commercial License (http://creativecommons.org/licenses/by-nc/4.0) which permits unrestricted non-commercial use, distribution, and reproduction in any medium, provided the original work is properly cited. 
CE for detecting dysplasia in UC, ${ }^{6}$ Kudo pit patterns showed a 92\% accuracy for differentiating between neoplastic and nonneoplastic lesions. However, in subsequent studies, Kudo pit patterns showed only 72\% to 73\% diagnostic accuracy for differentiating between neoplastic and non-neoplastic lesions in the colitis patients, ${ }^{11,12}$ which would be inappropriate to use as indicators for dysplasia in the clinical practice. The relative inaccuracy of pit patterns for predicting colitis-associated dysplasia may be associated with diffuse and long-standing inflammatory changes in both the dysplasia and surrounding mucosa. Recently, a Spanish group reported that the optic diagnosis with CE was highly accurate to rule out dysplasia. ${ }^{13}$ Nonetheless, in their study, Kudo pit patterns, protruded morphology, and loss of innominate lines showed just 62\%, 62\%, and 58\% of accuracy to predict dysplasia, respectively. ${ }^{13}$ As the predictive endoscopic features for dysplasia are relatively inaccurate, the decision over whether to perform biopsy during surveillance colonoscopy may vary across endoscopists. Furthermore, given that pedunculated dysplasias are easily recognizable and resectable, ${ }^{14-16}$ the decision on whether to take a targeted biopsy from nonpedunculated lesions is more important for endoscopists.

In this study, we used endoscopic images of target-biopsied lesions that had been confirmed as dysplastic or non-dysplastic to perform a survey of endoscopists, with the aim of evaluating the ability of endoscopists to discriminate dysplasia from nondysplasia.

\section{MATERIALS AND METHODS}

\section{Study design}

This survey-based study used photographs of target-biopsied lesions of UC patients who underwent surveillance colonoscopy for the detection of colitis-associated dysplasia or cancer. Ten expert endoscopists from four countries (Australia, Japan, Korea, and USA) participated in the survey and were asked to answer two questionnaires. One of these questionnaires consisted of questions about their experience as a gastrointestinal endoscopist such as the country where the endoscopist worked, the work period as a staff gastroenterologist, and the number of cases that the endoscopist had experienced. The other questionnaire consisted of questions about the endoscopic assessment of the provided lesion photographs. The key question was whether the participants agreed or disagreed that the suggested lesions were dysplastic. The respondents answered by choosing an answer from a 5-point Likert scale: strongly agree; agree; uncertain; disagree; strongly disagree. In addition to strongly agree or agree, the choice of uncertain was also defined as having intention to take targeted biopsies because the respondent could not exclude the possibility of dysplasia. The choices of disagree or strongly disagree were considered as the respondents having no intention to take biopsy. The endoscopists' assessments of the presence of dysplasia were therefore divided into suspected dys- plasia (strongly agree, agree, and uncertain) and non-dysplasia (disagree and strongly disagree). Strongly agree and strongly disagree were considered as highly confident endoscopic diagnoses of dysplasia and non-dysplastic lesions, respectively. Endoscopists were also asked to record the following for the lesions: Paris classification, ${ }^{5}$ surface ulceration, distinctness, and pit patterns. In addition, each endoscopist graded the endoscopic images according to the UC endoscopic index of severity (UCEIS). ${ }^{17}$ Age and gender of the patients, duration and extent of UC, history of primary sclerosing cholangitis, and previous history of dysplasia were retrieved from the institutional UC registry database. The participants were blinded to these data, which could be potentially associated with the risk of CRC or dysplasia. This study was approved by the Institutional Review Board of Asan Medical Center (IRB number: 2019-0037).

\section{Retrieval and selection of endoscopic images}

UC patients who underwent colonoscopy between August 2008 and September 2017 and with a disease duration of 8 years or longer or primary sclerosing cholangitis were retrieved from the institutional UC registry database. As histological diagnosis of dysplasia occasionally shows discrepancies across pathologists, ${ }^{18,19}$ and accurate histological diagnosis was essential for fulfilling the objectives of this study, only the following lesions were considered eligible: dysplastic or non-dysplastic lesions confirmed by biopsies taken during two or more separate surveillance colonoscopies with an interval of 6 months or longer, or by histological assessment of specimens from colectomy or endoscopic resection after initial forceps biopsybased diagnosis of dysplasia or cancer. The eligible lesions were reviewed by a board-certified pathologist (J.K.) specializing in gastrointestinal pathology. The endoscopic images of nondysplastic lesions were selected from the nonpedunculated or flat lesions confirmed as "negative for dysplasia" on at least two separate colonoscopic targeted biopsies taken over an interval of 6 months or longer. Endoscopic images of dysplastic lesions were selected from lesions confirmed as "colitis-associated dysplasia or cancer" on at least two separate colonoscopic targeted biopsies taken over an interval of 6 months or longer, or via endoscopic resection or colectomy specimens after diagnosis of dysplasia or cancer on one or more colonoscopic biopsies. The following lesions were excluded from this study: lesions diagnosed as dysplasia or cancer on the basis of the histology of a forceps-biopsy specimen taken at a single colonoscopy from a series of multiple repeated surveillance colonoscopies; those reported as indefinite for dysplasia; pedunculated or semipedunculated lesions (Paris 0-Ip); dysplasia or cancer located beyond the colitic segment; advanced CRC (Borrmann type I-IV or obstructive (RC), and squamous epithelial neoplasia on the anorectal junction. Lesions with low quality images were also excluded. Finally, 61 nonpedunculated target-biopsied lesions, including 27 non-dysplastic and 34 dysplastic lesions, were 
retrieved. Twenty lesions were photographed with both narrow band imaging (NBI) and indigo carmine-based CE, 26 were photographed under CE, and 14 under white light endoscopy (WLE) and NBI. Only one lesion was photographed under only WLE. All colonoscopic images were taken using high-definition colonoscopes (CF-HQ260 or CF-HQ290; Olympus Co., Tokyo, Japan) and were anonymized before being used for the survey.

\section{Outcomes}

The primary outcome of this survey was the hypothetical diagnostic performance of the endoscopists' intention to biopsy the lesions according to the provided photographs, and this was assessed using sensitivity, specificity, positive predictive value (PPV), negative predictive value (NPV), and accuracy. To calculate the diagnostic performance of the endoscopists' decision on whether to take biopsies or not, the hypothetical yield and accuracy of the target-biopsied specimen histology were set to $100 \%$. The secondary outcomes were interobserver agreements on the intention to take biopsy.

\section{Statistical analysis}

Sensitivity, specificity, PPV, NPV, and accuracy were calculated using MedCalc version 18.2.1 (MedCalc Software bvba, Ostend, Belgium). Fleiss kappa was calculated to evaluate interobserver agreement. The diagnostic performances between two different subgroups of endoscopists were compared using generalized estimating equations, and univariate analysis of endoscopic findings for the prediction of dysplasia was performed using a logistic regression model with generalized estimating equations, which was created with SAS version 9.4 (SAS Institute Inc., Cary, NC, USA).

\section{RESULTS}

\section{Characteristics of the UC patients and study participants}

The endoscopic images of 61 target-biopsied lesions from 56 UC patients were selected for this study (Fig. 1), and their demographic characteristics are summarized in Table 1. Details of the participant endoscopists are described in Table 2.

\section{Prediction of dysplasia based on the provided photo- graphs of target-biopsied lesions}

The endoscopists gave a mean ( \pm standard deviation) UCEIS of $0.8 \pm 0.7$ to the lesions in the provided images. The sensitivity, specificity, PPV, NPV, and accuracy according to the hypothetical histological results of the endoscopists' intention to take biopsies for target lesions were 88.2\% (95\% confidence interval [CI], $84.3 \%$ to $91.5 \%$ ), 34.8\% (95\% CI, 29.1\% to 40.8\%), 63.0\% (95\% CI, 60.8\% to 65.2\%), 70.2\% (95\% CI, 62.7\% to $76.6 \%$ ), and $64.6 \%$ (95\% CI, 60.7\% to 68.4\%), respectively. When limited to the high confidence assessments, the sensitivity, specificity, PPV, NPV, and accuracy were 93.0\% (95\% CI, 87.1\% to 96.7\%), 40.9\% (95\% CI, 26.3\% to 56.8\%), 82.1\% (95\% CI, 78.1\% to $85.5 \%$ ), $66.7 \%$ (95\% CI, $49.3 \%$ to $80.5 \%$ ), and $79.7 \%$ (95\% CI, $72.9 \%$ to $85.4 \%$ ), respectively. The sensitivity for photos with UCEIS 0 or 1 was superior to that for photos with UCEIS 2 or 3 , but duration of colitis, previous history of dysplasia, and presence of primary sclerosing cholangitis were not associated with the endoscopists' diagnostic performance (Table 3). Compared with those who had worked for less than 6 years as a staff endoscopist, those who had worked for 6 years or longer as a staff endoscopist showed significantly higher sensitivity in the prediction of dysplasia, but lower specificity. When the
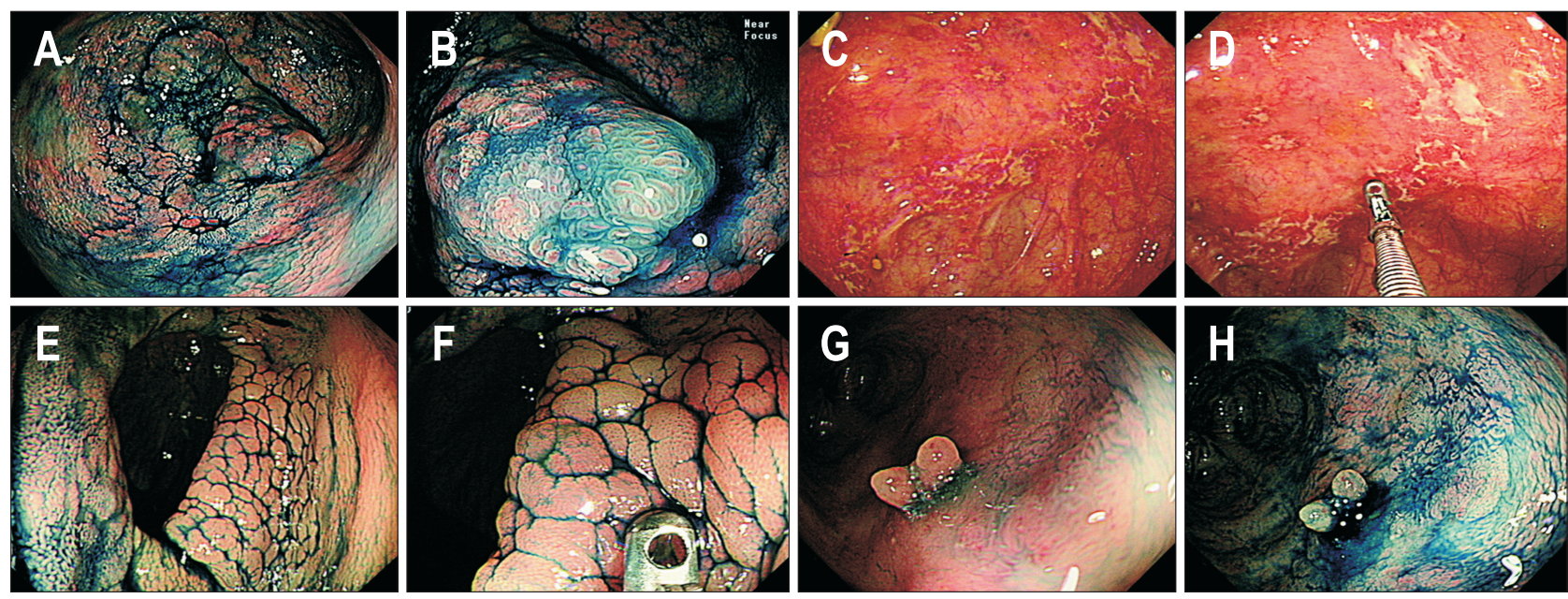

Fig. 1. (A, B) This lesion was assessed as dysplastic by all endoscopists. The final histology after colectomy was identified as an adenocarcinoma invading the proper muscle layer. (C, D) This lesion was assessed as "non-dysplastic" by four endoscopists, "dysplastic" by one endoscopist, and "uncertain but requiring targeted biopsies" by five endoscopists. Adenocarcinoma was identified by repeated forceps biopsies, and colectomy revealed poorly differentiated adenocarcinoma (T4N2M0). (E, F) This lesion was assessed as "non-dysplastic" by eight endoscopists, "dysplastic" by one endoscopist, and "uncertain but requiring targeted biopsies" by one endoscopist. Repeated forceps biopsies revealed no dysplasia. (G, H) This lesion was assessed as "non-dysplastic" by five endoscopists, "dysplastic" by three endoscopists, and "uncertain but requiring targeted biopsies" by two endoscopists. Repeated forceps biopsies revealed no dysplasia. 
endoscopists were sub-grouped according to their experience with dysplasia and UC patients, experienced endoscopists were less specific in the endoscopic prediction of dysplasia than lessexperienced endoscopists. The details are described in Table 3.

\section{Interobserver agreement on the intention to take biopsy}

The interobserver agreement on the intention to take biopsy was poor, with a Fleiss kappa of 0.169 (95\% CI, 0.132 to 0.206). However, the probability of dysplasia in the histology showed an increasing trend in association with an increasing proportion of endoscopists with intention to perform biopsy (Table 4).

\section{Associations between endoscopic findings and dysplasia diagnosis}

Of the 61 lesions, 15 lesions were represented by WLE+NBI or WLE images only, 26 by both CE and WLE images, and 20 by both NBI and CE images. When the lesions with WLE+NBI or WLE images were set as a reference group, the histologic diagnosis of dysplasia was not significantly associated with lesions having both CE and WLE images (odds ratio, 1.75; 95\% CI, 0.482 to $6.351 ; p=0.395$ ) or lesions with NBI and CE images (odds ratio, 3.5; 95\% CI, 0.856 to 14.303 ; $\mathrm{p}=0.081$ ).

of the three evaluated endoscopic characteristics (ulceration, distinctness of the borders, and pit pattern), neoplastic pit patterns (Kudo type III-V) were significantly predictive of dysplasia

Table 1. Characteristics of the UC Patients and the Target-Biopsied Lesions Selected for the Survey

\begin{tabular}{lc}
\multicolumn{1}{c}{ Variable } & Value \\
\hline No. of UC patients/target-biopsied lesions & $56 / 61$ \\
Histologic classification & $61(100)$ \\
Non-dysplastic lesion & $27(44.3)$ \\
Dysplasia or cancer & $34(55.7)$ \\
$\quad$ LGD & $18(29.5)$ \\
$\quad$ HGD or intramucosal cancer & $5(8.2)$ \\
$\quad$ Invasive cancer & $11(18.0)$ \\
Paris classification of biopsied lesions & \\
Is & $6(9.8)$ \\
II & $55(80.2)$ \\
Age at UC diagnosis, yr & $38.8 \pm 14.5$ \\
Age when target biopsy was taken, yr & $52.1 \pm 14.7$ \\
Duration of UC when target biopsy was taken, yr & $14.1 \pm 5.3$ \\
Extent of UC when target biopsy was taken & \\
Left-sided & $7(12.5)$ \\
Extensive & $49(87.5)$ \\
Primary sclerosing cholangitis & $7(12.5)$ \\
Previous history of dysplasia & $10(17.9)$ \\
\hline
\end{tabular}

Data are presented as number (\%) or mean \pm SD.

UC, ulcerative colitis; LGD, low-grade dysplasia; HGD, high-grade dysplasia. (odds ratio, 3.710; 95\% CI, 2.001 to 6.881, p<0.001) (Table 5) The endoscopists responded that $54.3 \%$ and $24.9 \%$ of lesions showed neoplastic or non-neoplastic pit patterns, respectively, and that the pit patterns of $20.8 \%$ of lesions were not assessible. The sensitivity, specificity, and accuracy of neoplastic pit patterns were $68.2 \%$ (95\% CI, 63.0\% to 73.2\%), 63.3\% (95\% CI, $57.3 \%$ to $69.1 \%$ ), and $66.1 \%$ (95\% CI, $62.2 \%$ to $69.8 \%$ ), respectively. The diagnostic performances of the other endoscopic features are described in Table 6.

\section{DISCUSSION}

In this study, we asked the study participants about their intention to take biopsies for a set of endoscopic images of nonpedunculated mostly non-polypoid lesions, and the primary outcome was the hypothetical diagnostic performance according to their intention to take biopsies from the given lesions. According to our data, if the respondents' intentions were to take biopsies for given lesions, the sensitivity, specificity, PPV, NPV, and accuracy were 88.2\%, 34.8\%, 63.0\%, 70.2\%, and $64.6 \%$, respectively. If the endoscopic prediction of dysplasia or non-dysplasia was made with a high confidence, the sensitivity, specificity, PPV, NPV, and accuracy were slightly improved at

Table 2. Characteristics of the Endoscopists Who Participated in This Survey

\begin{tabular}{lc}
\hline \multicolumn{1}{c}{ Variable } & $\begin{array}{c}\text { No. of } \\
\text { endoscopists }\end{array}$ \\
\hline Study participants & 10 \\
Country of the endoscopists & 6 \\
Korea & 1 \\
Japan & 2 \\
Australia & 1 \\
USA & \\
Work period as a staff endoscopist & 2 \\
$<6$ yr & 8 \\
$\geq 6$ yr & 5 \\
Volume of UC patients in the endoscopist's clinical practices & \\
$\leq 50$ cases/yr & 5 \\
$\geq 51$ cases/yr & \\
Surveillance colonoscopy per year & 5 \\
$\leq 10$ cases/yr & 5 \\
$\geq 11$ cases/yr & 5 \\
Volume of dysplasia in the endoscopist's center & \\
$2-10$ Cases till now & 5 \\
$\geq 11$ Cases till now & \\
Volume of dysplasia that the endoscopists experienced & \\
$2-10$ Cases till now & \\
\hline & \\
\hline
\end{tabular}

UC, ulcerative colitis. 
Table 3. The Endoscopists' Diagnostic Performance of the Nonpedunculated Dysplasia in the UC Patients

\begin{tabular}{|c|c|c|c|c|c|c|}
\hline Variable & $\begin{array}{l}\text { Sensitivity, } \\
\% \text { (95\% CI) }\end{array}$ & p-value & $\begin{array}{l}\text { Specificity, } \\
\% \text { (95\% CI) }\end{array}$ & p-value & $\begin{array}{l}\text { Accuracy, } \\
\% \text { (95\% CI) }\end{array}$ & p-value \\
\hline Overall & $88.2(84.3-91.5)$ & NA & $34.8(29.1-40.8)$ & NA & $64.6(60.7-68.4)$ & NA \\
\hline Overall-high confidence & $93.0(87.1-96.7)$ & NA & $40.9(26.3-56.8)$ & NA & 79.7 (72.9-85.4) & NA \\
\hline UCEIS & & 0.008 & & 0.473 & & 0.076 \\
\hline UCEIS 0-1 & $89.7(84.6-93.2)$ & & $35.7(27.0-45.4)$ & & $66.7(57.7-74.6)$ & \\
\hline UCEIS 2-3 & $73.3(57.2-85.0)$ & & $30.0(19.5-43.1)$ & & $48.6(31.2-66.3)$ & \\
\hline Duration of UC* & & 0.154 & & 0.405 & & 0.432 \\
\hline$<15 \mathrm{yr}$ & $91.1(83.9-95.2)$ & & $30.8(19.4-45.2)$ & & $67.7(54.8-78.4)$ & \\
\hline$\geq 15 \mathrm{yr}$ & $84.7(76.8-90.2)$ & & $38.0(28.6-48.4)$ & & $61.3(50.7-71.0)$ & \\
\hline Primary sclerosing cholangitis* & & 0.511 & & 0.069 & & 0.062 \\
\hline Absent & $87.6(82.0-91.6)$ & & $32.8(25.3-41.3)$ & & $62.2(53.4-70.3)$ & \\
\hline Present & $92.0(74.1-97.9)$ & & $60.0(32.1-82.6)$ & & $82.9(62.4-93.4)$ & \\
\hline Previous history of dysplasia* & & 0.549 & & 0.777 & & 0.493 \\
\hline Absent & $89.0(84.3-92.4)$ & & $35.5(27.3-44.6)$ & & $65.9(57.0-73.8)$ & \\
\hline Present & $84.0(57.8-95.3)$ & & $32.0(14.8-56.1)$ & & $58.0(36.4-76.9)$ & \\
\hline Region of the endoscopists & & 0.159 & & 0.198 & & 0.829 \\
\hline Western & $92.2(84.6-96.2)$ & & $30.9(21.7-41.9)$ & & $65.0(55.2-73.8)$ & \\
\hline Asian & $86.6(80.1-91.1)$ & & $36.5(28.5-45.3)$ & & $64.4(56.2-71.8)$ & \\
\hline Work duration as a staff endoscopist & & $<0.001$ & & $<0.001$ & & 0.796 \\
\hline$<6 \mathrm{yr}$ & $72.1(60.9-81.0)$ & & $57.4(44.9-69.0)$ & & $65.6(57.1-73.1)$ & \\
\hline$\geq 6 \mathrm{yr}$ & $92.3(88.1-95.1)$ & & $29.2(21.3-38.6)$ & & $64.3(55.0-72.7)$ & \\
\hline Endoscopist's experience in UC & & 0.069 & & $<0.001$ & & 0.044 \\
\hline$\geq 51$ patients/yr & $90.0(84.7-93.6)$ & & $27.4(20.0-36.4)$ & & $62.3(53.0-70.8)$ & \\
\hline $\begin{array}{l}\text { Endoscopist's experience in surveillance } \\
\text { colonoscopy }\end{array}$ & & 0.069 & & 0.002 & & 0.046 \\
\hline$\leq 10$ patients/yr & $86.5(80.5-90.8)$ & & $43.7(33.3-54.7)$ & & $67.5(59.4-74.7)$ & \\
\hline$\geq 11$ patients/yr & $90.0(84.7-93.6)$ & & $25.9(18.2-35.5)$ & & $61.6(52.1-70.3)$ & \\
\hline Endoscopist's experience in dysplasia & & 0.174 & & 0.022 & & 0.531 \\
\hline 2-10 Cases till now & $87.1(81.3-91.3)$ & & $37.0(29.1-45.7)$ & & $65.0(56.9-72.3)$ & \\
\hline$\geq 21$ Cases till now & $92.6(84.0-96.8)$ & & $25.9(16.7-38.0)$ & & $63.1(53.5-72.6)$ & \\
\hline Volume of dysplasia in the endoscopist's center & & 0.057 & & 0.035 & & 0.468 \\
\hline 2-10 Cases till now & $85.9(79.4-90.6)$ & & 40.0 (30.9-49.9) & & $65.6(57.4-72.9)$ & \\
\hline$\geq 11$ Cases till now & $90.6(85.2-94.2)$ & & $29.6(21.3-39.6)$ & & $63.6(54.3-72.0)$ & \\
\hline
\end{tabular}

UC, ulcerative colitis; CI, confidence interval; NA, not applicable; UCEIS, UC endoscopic index of severity.

*Information about the duration of UC, primary sclerosing cholangitis, and previous history of dysplasia was not provided to the endoscopists who participated in the survey.

Table 4. Probability of Dysplasia in Association with the Number of Endoscopists Who Expressed Their Intention to Perform a Biopsy of the Provided Lesions

\begin{tabular}{|c|c|c|c|c|c|c|c|c|c|}
\hline No. of endoscopists with intention to take biopsy & 2 & 3 & 4 & 5 & 6 & 7 & 8 & 9 & 10 \\
\hline No. of endoscopists without intention to take biopsy & 8 & 7 & 6 & 5 & 4 & 3 & 2 & 1 & 0 \\
\hline Dysplasia (total, $\mathrm{n}=34$ ) & 0 & 0 & 0 & 1 & 1 & 3 & 7 & 8 & 14 \\
\hline Non-dysplasia (total, $\mathrm{n}=27$ ) & 2 & 1 & 2 & 3 & 4 & 5 & 4 & 5 & 1 \\
\hline Probability of dysplasia, \% & 0 & 0 & 0 & 25 & 20 & 37.5 & 63.6 & 61.5 & 93.3 \\
\hline
\end{tabular}


93.0\%, 40.9\%, 82.1\%, 66.7\%, and 79.7\%, respectively. Meanwhile, the sensitivity was higher in the lesions with UCEIS 0 or 1 than in the lesions with UCEIS 2 or 3 in our data, and this supports that surveillance colonoscopy should be performed after achieving endoscopic remission, if possible. The diagnostic performance profile for dysplasia in this study seems different to that in a recent study of prospective multicenter data from Spain. ${ }^{13}$ This previous study analyzed 596 lesions (214 protruding and 382 flat lesions) located in the colitic area and reported that the sensitivity, specificity, PPV, NPV, and accuracy of optical diagnosis for dysplasia were 70\%, 90\%, 58\%, 94\%, and $87.4 \%$, respectively. In comparison, our study showed higher sensitivity but lower specificity for prediction of dysplasia. Differences in the proportion of Paris II morphology (80.5\% in our study and 64\% in the Spanish study) and the study design (retrospective in ours vs prospective in the Spanish study) might be associated with the differences in the results between the two studies. As our study participants assessed the lesions using only the provided images, without any clinical information, our survey results cannot reflect the real-life diagnostic performance of dysplasia surveillance in UC patients. Nonetheless, the current study suggests that endoscopic prediction of dysplasia may not be sufficiently accurate to allow skipping of targeted biopsies from nonpedunculated or non-polypoid lesions detected during surveillance colonoscopy in UC patients.

The poor interobserver agreement on the intention to take bi-

Table 5. Association of Dysplasia with Endoscopic Variables of the Target-Biopsied Lesions

\begin{tabular}{lcc}
\hline \multicolumn{1}{c}{ Endoscopic findings } & OR (95\% CI) & p-value \\
\hline Ulceration & $1.772(0.709-4.428)$ & 0.221 \\
Ulcer (+) & 1 (reference) & \\
Ulcer (-) & & \\
Border & 1.739 (0.918-3.293) & 0.090 \\
Distinct or assumable & 1 (reference) & \\
Indistinct border & & \\
Pit pattern & 3.710 (2.001-6.881) & $<0.001$ \\
Neoplastic & 1 (reference) & \\
Non-neoplastic/not assessable & & \\
\hline
\end{tabular}

$\mathrm{OR}$, odds ratio; $\mathrm{CI}$, confidence interval. opsy in this study may be associated with a lack of well-defined endoscopic characteristics of dysplasia, which could otherwise be used as indicators for taking targeted biopsies during surveillance. However, those lesions that showed higher concordance rates over whether the endoscopists had intention to take biopsy were more likely to be dysplasia. This means that there may be common characteristics that are not yet clearly defined, but that are useful for discriminating between dysplasia and nondysplasia. A neoplastic pit pattern is considered an excellent endoscopic feature for predicting sporadic colorectal neoplasia, ${ }^{20}$ and its diagnostic performance for predicting colitis-associated dysplasia was investigated in several previous studies. In an early randomized controlled trial comparing panchromoendoscopy with white light colonoscopy for surveillance in long-standing UC patients, the sensitivity, specificity, and accuracy of neoplastic pit patterns for predicting dysplasia were 94\%, 93\%, and 92\%, respectively. ${ }^{6}$ However, a subsequent study stated only a limited role of neoplastic pit patterns for predicting colitisassociated dysplasia in long-standing UC. ${ }^{12}$ Recently, Bisschops et al. ${ }^{11}$ used lesions retrieved from a randomized controlled trial comparing high-definition CE with NBI for the surveillance of colitis-associated dysplasia, and analyzed the diagnostic performance of pit patterns for predicting dysplasia. Carefully selected images of clearly visible lesions with visible pits were used in their study, and the sensitivity, specificity, and accuracy of neoplastic pit patterns for predicting dysplasia were 77\%, 69\%, and 72\%, respectively. ${ }^{11}$ The aforementioned Spanish multicenter prospective study found the sensitivity, specificity, and accuracy of neoplastic pit patterns for predicting dysplasia to be $32 \%$, $92 \%$, and $62 \%$, respectively. ${ }^{13}$ In the present study, the sensitivity, specificity, and accuracy of neoplastic pit patterns were $68.2 \%$, $63.3 \%$, and $66.1 \%$, respectively. Therefore, differing from the earliest study, the pit patterns of suspected dysplasia showed only limited diagnostic performance in recent studies, including ours.

Protruding lesions (Paris Ip and Is) are easily recognizable during surveillance colonoscopy of colitic patients, and in the Spanish study, a polypoid appearance itself showed sensitivity of $56 \%$ and specificity of $68 \%$ for predicting dysplasia. However, the recognition of non-polypoid dysplasia is quite challenging. Several signs related to non-polypoid dysplasia were described in a recent paper, including interruption of innominate lines, wall deformity, spontaneous friability, shallow ulcer,

Table 6. Performance of Endoscopic Features in the Diagnosis of Nonpedunculated Dysplasia or Cancer in Ulcerative Colitis Patients

\begin{tabular}{|c|c|c|c|c|c|}
\hline Findings & $\begin{array}{l}\text { Sensitivity, } \\
\%(95 \% \text { CI })\end{array}$ & $\begin{array}{l}\text { Specificity, } \\
\%(95 \% \text { CI })\end{array}$ & $\begin{array}{c}\text { PPV, } \\
\%(95 \% \mathrm{CI})\end{array}$ & $\begin{array}{c}\text { NPV, } \\
\%(95 \% \text { CI })\end{array}$ & $\begin{array}{l}\text { Accuracy, } \\
\%(95 \% \text { CI })\end{array}$ \\
\hline Ulceration (vs no ulceration) & $20.9(16.7-25.6)$ & $87.0(82.4-90.8)$ & $67.0(58.3-74.6)$ & $46.6(44.9-48.4)$ & $50.2(46.1-54.2)$ \\
\hline Distinct border (vs indistinct border) & $74.1(69.1-78.7)$ & $37.8(32.0-43.9)$ & $60.0(57.3-62.7)$ & $53.7(47.8-59.5)$ & $58.0(54.0-62.0)$ \\
\hline $\begin{array}{l}\text { Neoplastic pit pattern (vs non-neoplastic } \\
\text { or unassessable pit patterns) }\end{array}$ & $68.2(63.0-73.2)$ & $63.3(57.3-69.1)$ & $70.1(66.4-73.6)$ & $61.3(56.9-65.5)$ & $66.1(62.2-69.8)$ \\
\hline
\end{tabular}

PPV, positive predictive value; NPV, negative predictive value; CI, confidence interval. 
velvety appearance, villous mucosa, slight discoloration, and "red in blue" sign during CE. ${ }^{21}$ Most of these descriptors seem difficult to standardize, although the interobserver agreements for these features have not yet been investigated. Moreover, little is known about the diagnostic performance of these descriptors for predicting dysplasia, although the sensitivity and specificity of interrupted innominate lines were both reported as 56\% in one study. ${ }^{13}$

Interestingly, we found that endoscopists with longer clinical experience showed higher sensitivity and lower specificity for predicting dysplasia than those with shorter clinical experience. Similarly, endoscopists with a high volume of experience in UC and surveillance colonoscopy showed lower specificity and a trend towards higher sensitivity for prediction of dysplasia than the endoscopists with a low volume of experience in UC and surveillance colonoscopy. Endoscopists with more experience in dysplasia or belonging to centers with a higher volume of dysplasia patients showed lower specificity than the others. These findings may imply that endoscopists with more experience have a tendency to sacrifice accuracy, not to miss a dysplasia mimicking an inflammatory lesion.

A targeted biopsy strategy is one of the most remarkable changes in the recent guidelines on surveillance colonoscopy of UC patients at risk of CRC. ${ }^{3,10}$ Improved recognition of potential dysplastic lesions under CE or image-enhanced endoscopy can enhance the effectiveness of a targeted biopsy strategy. ${ }^{10,22}$ However, the endoscopic discrimination of dysplasia from nondysplasia is also necessary to maximize the efficiency of a targeted biopsy strategy. Currently, the absence of neoplastic pit patterns and endoscopic features related to non-polypoid dysplasia cannot exclude the possibility of dysplasia during surveillance of long-standing UC patients. As a matter of course, endoscopists take biopsies from any suspected dysplastic lesions encountered during surveillance colonoscopy, unless they can precisely exclude the possibility of dysplasia on the basis of the endoscopic findings. Therefore, even if targeted biopsy surveillance colonoscopy is performed, unreliable endoscopic prediction of dysplasia will make surveillance colonoscopy less efficient in real practice.

This study is subject to several limitations. First, the endoscopists' assessments of the possibility of dysplasia and their characterizations of the provided lesions were performed on the basis of the provided endoscopic images, and they were not based on real-time endoscopic observation. The photographs provided for this survey did not include photographs of abnormal lesions, which could more easily be identified as being nondysplastic and were not biopsied during colonoscopy. Therefore, the selected photos might have been more confusing for the respondents than real-life surveillance colonoscopy, and the performance of the study participants for diagnosing dysplasia might have been underestimated compared with their real diagnostic performance for dysplasia. Second, the selected photos

of potential dysplasia were not taken using a standardized protocol. Therefore, CE images were not available for some lesions, despite these being recommended by the recent guidelines for examination of potential dysplastic lesions during surveillance colonoscopy. ${ }^{10}$ Third, the results of the current survey may have been influenced by the endoscopist's concern about, or experience of, dysplasia or colitic cancer having an atypical endoscopic appearance; for example, dysplasia or colitic cancer without neoplastic pit patterns or mimicking inflammatory change. Our survey did not include questions related to the endoscopists' concerns about missing atypical dysplasia during surveillance.

In conclusion, the endoscopists' diagnostic performance for potentially dysplastic lesions in UC patients based on their intentions to take biopsies was found to be suboptimal. The diagnostic performance of endoscopic features known to be associated with dysplasia should be more extensively investigated and indicators for taking targeted biopsies should be established to provide clear guidance on surveillance colonoscopy for longstanding UC patients.

\section{CONFLICTS OF INTEREST}

No potential conflict of interest relevant to this article was reported.

\section{ACKNOWLEDGEMENTS}

D.H.Y., S.J., J.M.C., J.S.B., H.W.K., H.G.K., and F.M. are the members of a research group named Asian Pacific Research in Endoscopy (AsPiRE). This study was initiated at a meeting of AsPiRE.

\section{AUTHOR CONTRIBUTIONS}

Planning the study: D.H.Y., S.J., F.M., J.M.C., J.S.B., H.W.K., H.G.K., B.D.Y., J.K., S.K.Y. Conducting the study: D.H.Y., S.J., F.M., J.M.C., J.S.B., H.W.K., H.G.K., S.F., Y.X.K., J.Y.Y., M.S.K., J.K. Collecting data: D.H.Y., S.J., F.M., J.M.C., J.S.B., H.W.K., H.G.K., S.F., Y.X.K., J.Y.Y., M.S.K., B.D.Y., S.K.Y. Interpreting data: D.H.Y., B.D.Y., S.K.Y., J.K. Drafting the manuscript: D.H.Y. Review of pathology: J.K. Approval of the final draft: all authors.

\section{ORCID}

Dong-Hoon Yang

Sneha John

Fujishiro Mitsuhiro

Jae Myung Cha

Jeong-Sik Byeon

Hyung Wook Kim

Hyun Gun Kim https://orcid.org/0000-0001-7756-2704 https://orcid.org/0000-0001-9332-3826 https://orcid.org/0000-0002-4074-1140 https://orcid.org/0000-0001-9403-230X https://orcid.org/0000-0002-9793-6379 https://orcid.org/0000-0002-2444-0591 https://orcid.org/0000-0001-7545-4638 


\section{Shai Friedland \\ Yon Xian Koh \\ Jin-Young Yoon \\ Min-Seob Kwak \\ Byong Duk Ye \\ Jihun Kim \\ Suk-Kyun Yang}

\section{REFERENCES}

1. Jess T, Rungoe C, Peyrin-Biroulet L. Risk of colorectal cancer in patients with ulcerative colitis: a meta-analysis of populationbased cohort studies. Clin Gastroenterol Hepatol 2012;10:639-645.

2. Colman RJ, Rubin DT. Histological inflammation increases the risk of colorectal neoplasia in ulcerative colitis: a systematic review. Intest Res 2016;14:202-210.

3. Annese V, Daperno M, Rutter MD, et al. European evidence based consensus for endoscopy in inflammatory bowel disease. J Crohns Colitis 2013;7:982-1018.

4. Farraye FA, Odze RD, Eaden J, et al. AGA medical position statement on the diagnosis and management of colorectal neoplasia in inflammatory bowel disease. Gastroenterology 2010;138:738-745.

5. American Society for Gastrointestinal Endoscopy Standards of Practice Committee, Shergill AK, Lightdale JR, et al. The role of endoscopy in inflammatory bowel disease. Gastrointest Endosc 2015;81:1101-1121.

6. Kiesslich R, Fritsch J, Holtmann M, et al. Methylene blue-aided chromoendoscopy for the detection of intraepithelial neoplasia and colon cancer in ulcerative colitis. Gastroenterology 2003;124:880-888.

7. Matsumoto T, Nakamura S, Jo Y, Yao T, Iida M. Chromoscopy might improve diagnostic accuracy in cancer surveillance for ulcerative colitis. Am J Gastroenterol 2003;98:1827-1833.

8. Rutter MD, Saunders BP, Schofield G, Forbes A, Price AB, Talbot IC. Pancolonic indigo carmine dye spraying for the detection of dysplasia in ulcerative colitis. Gut 2004;53:256-260.

9. Marion JF, Waye JD, Present DH, et al. Chromoendoscopy-targeted biopsies are superior to standard colonoscopic surveillance for detecting dysplasia in inflammatory bowel disease patients: a prospective endoscopic trial. Am J Gastroenterol 2008;103:2342-2349.

10. Laine L, Kaltenbach T, Barkun A, et al. SCENIC international consensus statement on surveillance and management of dysplasia in inflammatory bowel disease. Gastroenterology 2015;148:639-651.

11. Bisschops R, Bessissow T, Dekker E, et al. Pit pattern analysis with high-definition chromoendoscopy and narrow-band imaging for optical diagnosis of dysplasia in patients with ulcerative colitis. Gastrointest Endosc 2017;86:1100-1106.

12. Hata K, Watanabe T, Motoi T, Nagawa H. Pitfalls of pit pattern diagnosis in ulcerative colitis-associated dysplasia. Gastroenterology 2004;126:374-376.

13. Carballal S, Maisterra S, López-Serrano A, et al. Real-life chromoendoscopy for neoplasia detection and characterisation in longstanding IBD. Gut 2018;67:70-78.

14. Rubin PH, Friedman S, Harpaz N, et al. Colonoscopic polypectomy in chronic colitis: conservative management after endoscopic resection of dysplastic polyps. Gastroenterology 1999;117:1295-1300.

15. Engelsgjerd M, Farraye FA, Odze RD. Polypectomy may be adequate treatment for adenoma-like dysplastic lesions in chronic ulcerative colitis. Gastroenterology 1999;117:1288-1294.

16. Wanders LK, Dekker E, Pullens B, Bassett P, Travis SP, East JE. Cancer risk after resection of polypoid dysplasia in patients with longstanding ulcerative colitis: a meta-analysis. Clin Gastroenterol Hepatol 2014;12:756-764.

17. Travis SP, Schnell D, Krzeski P, et al. Developing an instrument to assess the endoscopic severity of ulcerative colitis: the Ulcerative Colitis Endoscopic Index of Severity (UCEIS). Gut 2012;61:535-542.

18. Allende D, Elmessiry M, Hao W, et al. Inter-observer and intraobserver variability in the diagnosis of dysplasia in patients with inflammatory bowel disease: correlation of pathological and endoscopic findings. Colorectal Dis 2014;16:710-718.

19. Ullman T, Odze R, Farraye FA. Diagnosis and management of dysplasia in patients with ulcerative colitis and Crohn's disease of the colon. Inflamm Bowel Dis 2009;15:630-638.

20. Wada Y, Kashida H, Kudo SE, Misawa M, Ikehara N, Hamatani S. Diagnostic accuracy of pit pattern and vascular pattern analyses in colorectal lesions. Dig Endosc 2010;22:192-199.

21. Soetikno R, East J, Suzuki N, et al. Endoscopic submucosal dissection for nonpolypoid colorectal dysplasia in patients with inflammatory bowel disease: in medias res. Gastrointest Endosc 2018;87:1085-1094.

22. Iacucci M, Kaplan GG, Panaccione R, et al. A randomized trial comparing high definition colonoscopy alone with high definition dye spraying and electronic virtual chromoendoscopy for detection of colonic neoplastic lesions during IBD surveillance colonoscopy. Am J Gastroenterol 2018;113:225-234. 